UWE KÜHL

\title{
LE DÉBAT SUR LE SOCIALISME MUNICIPAL EN ALLEMAGNE AVANT 1914 ET LA MUNICIPALISATION DE L'ÉLECTRICITÉ ${ }^{1}$
}

\author{
Introduction
}

Dans l'historiographie nationale aussi bien qu'internationale, l'Empire allemand avant 1914 passe pour un pays où le soi-disant "socialisme municipal"" a trouvé une expansion et une propagation tout à fait exceptionnelles. Le SM est considéré ici comme corrélé au "socialisme d'État", un courant de politique économique qui s'est développé au sein du "Verein für Socialpolitik" à partir du début des années 1870. Reposant sur des traditions plus anciennes, l'accueil positif réservé aux interventions de l'État rendait possible que les villes en croissance rapide du fait du processus d'urbanisation exploitassent de plus en plus en régie municipale des entreprises commerciales au service des habitants. Le SM s'identifiait aux efforts déployés, avant tout dans les villes, "pour fournir des services répondant aux besoins quotidiens des habitants par la municipalisation des entreprises monopolistiques privées de la ville dans le domaine des services et du transport urbain et par la création d'autres établissements à caractère hygiénique, sanitaire, social et culturel"

La rapide réception du concept de SM en Allemagne est attribuée communément aux activités des soi-disant "socialistes de la chaire", ce groupe d'économistes et de sociologues qui s'étaient rassemblés dans le "Verein für Socialpolitik" et qui plaidaient de différentes manières pour des réformes sociales". A vrai

\footnotetext{
${ }^{1}$ Je remercie Jean Lorcin d'avoir bien voulu relire cet article.

${ }^{2}$ Désormais abrégé SM.

${ }^{3}$ Wolfgang R. KRABBE, Munizipalsozialismus und Interventionsstaat. Die Ausbreitung der städtischen Leistungsverwaltung im Kaiserreich, dans: Geschichte in Wissenschaft und Unterricht 30 (1979) p. 265-283, ici 267sv.: "durch Kommunalisierung privater städtischer Monopolbetriebe des Versorgungs- und Verkehrsbereichs und durch Neuetablierung anderer Anstalten stadthygienischer, gesundheitlicher, sozialer und kultureller Art für die Daseinsvorsorge der Gemeindeeinwohner Leistungen zu erbringen." [traduction U. K.]

"Pour le "Verein für Socialpolitik" voir Franz BOESE, Geschichte des Vereins für Sozialpolitik 1872-1932, Berlin 1939 (Schriften des Vereins für Socialpolitik [ci-après abrégé SVfSP], 188); Dieter LINDENLAUB, Richtungskämpfe im Verein für Sozialpolitik, Wiesbaden 1967 (VJS für Sozial- und Wirtschaftsgeschichte, Beihefte 52, 53); Irmela GORGES, Sozialforschung in Deutschland 1872-1914, Königstein, Ts. 1980 (Schriften des Wissenschaftszentrums Berlin, 14).
} 
dire, ce rapport jusqu'à présent n'est pas encore suffisamment prouvé. Certes, on recourt toujours aux grandes enquêtes du "Verein für Socialpolitik" pour présenter la propagation des entreprises municipales dans le Reich. Pourtant, celles-ci ne datent que des années après la fin du siècle. La municipalisation des établissements commerciaux, soit par transformation d'entreprises jusqu'ici privées, soit par fondation directe en régie, a commencé beaucoup plus tôt en Allemagne. Ce furent en premier lieu les usines à gaz qui furent affectées par la transformation en régie municipale ${ }^{5}$. Par contre, les usines électriques, dans les grandes villes allemandes, furent dès le début exploitées dans leur grande majorité comme entreprises municipales. En cela le développement en Allemagne se distingue fondamentalement de celui, par exemple, en France ${ }^{6}$.

Cette contribution vise à tirer au clair les questions suivantes: d'un côté la réception du concept de SM, qui pose en particulier la question du rôle des socialistes de la chaire; d'autre part, on se demandera pour quelle raison les grandes villes allemandes ont mis en régie directe leurs usines électriques dès le début. Autre chose : Etait-ce vraiment l'idée d'un SM ou d'autres considérations qui ont mené à cette décision?

Depuis peu, les chercheurs se sont demandé les raisons décisives de ces investissements communaux sous l'Empire, les usines électriques étant des investissements extrêmement volumineux. En retournant aux débats contemporains, on cite trois hypothèses qui auraient déterminé les décisions municipales d'investissement. Il y a en premier lieu la thèse d'une soi-disant stratégie de la détente fiscale prévisionnelle, c'est-à-dire qu'on acquiert et agrandit des entreprises municipales pour réaliser des rentrées supplémentaires non sans importance pour le budget communal. Mais les investissements municipaux pouvaient être aussi une réponse aux besoins des citadins en services nouveaux, voire étendus. La réponse à ces besoins dépendait de la demande effective existant dans les villes, laquelle à son tour était déterminée par les revenus disponibles de la population. Enfin, on peut interpréter les investissements municipaux comme une stratégie de politique sociale qui était fondée sur une idéologie et pour laquelle, au cours du XIX ${ }^{\mathfrak{c}}$ siècle, le concept de SM, venu d'Angleterre, s'était aussi très vite implanté en Allemagne?

${ }^{5}$ Voir Hans-Dieter BRUNCKHORST, Kommunalisierung im 19. Jahrhundert dargestellt am Beispiel der Gaswirtschaft in Deutschland, München 1978.

${ }^{6}$ Pour une comparaison des débuts de l'électrification des grandes villes en France et en Allemagne voir Uwe KÜHL, Anfänge städtischer Elektrifizierung in Deutschland und Frankreich, dans: Dieter SCHOTT (éd.), Energie und Stadt in Europa, Stuttgart 1997 (Vierteljahrschrift für Sozial- und Wirtschaftsgeschichte, Beiheft 135) p. 129-139.

${ }^{7}$ Voir Richard H. TILLY, Städtewachstum, Kommunalfinanzen und Munizipalsozialismus in der deutschen Industrialisierung: eine vergleichende Perspektive 1870-1913, dans: Jürgen REULECKE (éd.), Die Stadt als Dienstleistungszentrum. Beiträge zur Geschichte der "Sozialstadt" in Deutschland im 19. und frühen 20. Jahrhundert, St. Katharinen 1995, p. 125-152; ID., Investitionen im Deutschen Kaiserreich, dans: Karl H. KaUFHOLD (éd.), Investitionen der Städte im 
Pour notre problème n'entrent ici en ligne de compte que la première et la troisième thèse comme explication. En effet, la question de savoir si une usine électrique devait être exploitée en régie ou privatisée ne jouait aucun rôle pour répondre à une demande effective. A mon avis, on peut expliquer l'option de la forme de propriété d'après la politique sociale i.e. donc l'idéologie du SM, ou alors elle était motivée par la politique financière, développer de nouvelles rentrées pour la municipalité, ce qui lui donnait la possibilité de renoncer aux augmentations d'impôts directs. La constitution politique des villes allemandes avec un droit de vote extrêmement censitaire offrait en effet aux classes supérieures de bonnes occasions d'échapper aux augmentations d'impôts grâce à des entreprises municipales. Jusqu'à présent les études économétriques concernant cette question ont cependant montré qu'une explication monocausale de l'ampleur des investissements municipaux n'est pas possible.

Dès lors, je vais esquisser tout d'abord la réception du concept de SM et son interprétation en Allemagne. Après cela, je présenterai le mode et l'expansion de la régie directe dans le domaine de l'approvisionnement en électricité. Enfin, j'essaierai d'arriver à une conclusion qualitativement fondée sur les raisons de la régie municipale.

\section{Le concept de socialisme municipal en Allemagne avant 1914}

La réception du concept de SM, "municipal socialism", "Munizipalsozialismus" fut facile en Allemagne, car il y avait été fait un travail idéologique préparatoire avec la notion et le concept comparables de "Staatssozialismus", "state socialism", socialisme d'État. Les avocats d'une intervention de l'État pour résoudre la soi-disant "question ouvrière" ou "question sociale", comme d'une plus forte intervention dans l'économie capitaliste ébranlée par des crises, s'étaient groupés dans le "Verein für Socialpolitik" à partir de 1872. Ce groupe de savants, hauts fonctionnaires et journalistes essayait d'organiser un contrepoids au courant libéral encore prépondérant en ce temps. Parmi eux, les professeurs d'économie politique jouaient un rôle éminent, ce qui fit donner au groupe l'étiquette injurieuse de "Kathedersozialisten" par la presse "libérale manchestérienne".

Le concept de SM est considéré communément comme une création de la

19. und 20. Jahrhundert, Köln,Weimar,Wien 1997, p. 39-59; ID., Kommunalfinanzen und -investionen im Deutschen Kaiserreich, 1870-1914: Quantifizierungsansätze, dans: Hans-Jürgen GERHARD (éd.), Struktur und Dimension. Festschrift f. Karl H. Kaufhold, Bd. 2, Stuttgart 1997, p. 134-166. Michael HÜHNER, Kommunalfinanzen, Kommunalunternehmen und Kommunalpolitik im Deutschen Kaiserreich, Münster 1998 (Münsteraner Beiträge zur Cliometrie und quantitativen Wirtschaftsgeschichte, 6 ). 
"London Fabian Society". Ce groupe d'intellectuels anglais réunis autour de Sidney et Beatrice Webb et de George Bernard Shaw propageait un socialisme réformiste. Outre une imposition équitable, c'était la nationalisation des chemins de fer et des mines de charbon, ainsi que la municipalisation des services urbains les plus importants qui devaient aplanir les voies du socialisme ${ }^{8}$. Cette dernière n'était en Angleterre ni un phénomène nouveau ni même une exigence politique qui aurait été limitée au camp socialiste. La propriété municipale des services des eaux, des usines à gaz ou des tramways était absolument normale en Angleterre longtemps avant que les Fabiens ne formulassent de telles revendications. L'attraction du programme de politique communale des Fabiens s'explique probablement avant tout par l'état complètement sous-développé et désolant de la municipalité londonienne. Avant tout aussi, en comparaison avec les autres grandes villes anglaises et écossaises, il y avait ici un sérieux besoin de réformes. Mais la demande spécifique de socialisme municipal ne provenait en aucune façon exclusivement du cercle de la "Fabian Society", mais avaient déjà été soutenues par des hommes politiques communaux libéraux. Mais il ne faut pas sous-estimer la dynamique que reçut le programme des "progressistes" de la propagande correspondante des Fabiens. C'est ainsi que furent publiés en juillet 1889 les "Facts for Londoners", qui étaient pleins de matériel statistique sur l'état de la ville et de propositions de réformes sur la base du programme fabien. Cet ouvrage "was the raw material of Municipal Socialism, and from this time forth the Society recognised that the municipalisation of monopolies was a genuine part of the Socialist programme, that the transfer from private exploiters to public management at the start, and ultimately by the amortisation of the loans to public ownership, actually was pro tanto the transfer from private to public ownership of land and capital, as demanded by Socialists" ${ }^{10}$. Le "London Programme" publié par Sidney Webb en 1891 fut en quelque sorte le programme de base du SM. Le London County Council fondé en 1888 comme nouvelle autorité de self-government fut la scène des débats politiques sur ce programme. Alors que dans les villes de province la municipalisation était regardée comme une question purement formelle sans importance politique ou sociale, elle constituait à Londres un vrai champ de bataille des partis politiques. Les revendications des socialistes provoquaient chez les individualistes une opposition vraiment fondamentale: "and the tramways of London were held as a trench in the world-wide conflict between Socialism and its enemies, whose capture was hailed as an omen of progress by one side, and by the other deplored as the presage of defeat"11.

\footnotetext{
${ }^{8}$ Voir A. M. MCBRIAR, Fabian Socialism and English Politics 1884-1918, Cambridge 1962; Edward R. PEASE, The History of the Fabian Society, London ${ }^{3} 1963$; Edgar REICHEL, Der Sozialismus der Fabier, Heidelberg 1947; KRABBE, Munizipalismus und Interventionsstaat 1979.

${ }^{9}$ Fabian Tract No. 8.

${ }^{10}$ PEASE, The History of Fabian Society 1963 , p. 81 .

${ }^{11}$ Ibid., p. 82.
} 
La programmatique du SM aussi bien que les préoccupations des socialistes français pour les communes ne furent accueillies qu'avec hésitation dans les milieux professionnels germanophones. Il était caractéristique que ce fût un haut fonctionnaire municipal de Vienne qui présentât pour la première fois la théorie du SM de Sidney Webb ${ }^{12}$. Le public allemand s'intéressant au développement anglais put en prendre connaissance au plus tard à partir de 1897. Dans cette année non seulement parut pour la première fois une collection des "Fabian Essays" en allemand ${ }^{13}$, mais aussi en même temps l'expert de la SPD en politique communale, Hugo Lindemann, introduisit vraiment en Allemagne le concept de SM avec son livre sur l'administration urbaine en Angleterre ${ }^{14}$. Le livre suscita un tel intérêt qu'il parut dès 1906 une seconde édition. Son auteur, qui était devenu une autorité réputée dans ce domaine avec d'autres études de base sur l'administration municipale après la fin du siècle, pouvait revendiquer avec raison que son livre "(ait) apporté sa contribution à faire naître et accélérer le développement moderne en Allemagne" 15 . Le succès de ce livre était sans doute fondé sur ce qu'il visait à deux cibles très différentes: réformateurs sociaux bourgeois et représentants de la tendance révisionniste du mouvement ouvrier de l'autre. Malgré la puissance du développement de l'Allemagne, la Grande-Bretagne restait le pays le plus avancé économiquement et socialement. Elle était encore un modèle pour une grande partie de la bourgeoisie d'Allemagne, qui découvrait dans son développement son propre avenir ${ }^{16}$. Aussi l'administration municipale anglaise était-elle un objet de réflexion extrêmement intéressant et important pour les politiciens communaux allemands ${ }^{17}$. Lindemann présentait en détail les performances des villes anglaises qu'exigeait le rapide processus d'urbanisation. La présentation riche en matières avait suscité un intérêt d'autant plus grand qu'elle donnait la possibilité de bien mesurer son propre degré d'évolution. Même si un socialiste élevait des revendications étendues pour une activité économique communale, il n'y avait pas de barrière idéologique à surmonter, vu que la patrie

${ }^{12}$ Victor MATAJA, Städtische Socialpolitik, dans: Zeitschrift für Volkswirtschaft, Socialpolitik und Verwaltung 3 (1894) p. 519-597, évoquant le "Gemeindesocialismus" comme l'influence exercée sur les conditions de travail par les municipalités par analogie au socialisme d'État.

${ }^{13}$ Englische Socialreformer. Eine Sammlung "Fabian Essays", s.l.d.d. M. GRUNWALD, Leipzig 1897; ce volume parut dans Bibliothek für Socialwissenschaft, édité entre autres par Werner Sombart.

${ }^{14} \mathrm{H}$. LINDEMANN, Städteverwaltung und Munizipal-Sozialismus in England, Stuttgart 1897.

${ }^{15}$ LINDEMANN, Städteverwaltung und Munizipal-Sozialismus ${ }^{2} 1906$, p. VII: "sein Teil dazu beigetragen (habe), die moderne Entwicklung der deutschen Städteverwaltung auszulösen und zu beschleunigen."

${ }^{16}$ Le regard vers l'Angleterre se dirigeait avant tout au développement économique et technique.

${ }^{17}$ Le self-government anglais comme conçu par le juriste libéral Gneist avait eu un vif écho en Allemagne; voir Heinrich HEFFTER, Die deutsche Selbstverwaltung im 19. Jahrhundert, Stuttgart 1950. 
de la libre concurrence montrait précisément cette voie ${ }^{18}$. Pour Lindemann luimême, ses camarades auraient dû être ses principaux destinataires.

Jusqu'à présent, le SPD avait à peine fixé son attention sur les communes et la politique communale. Certes, le parti revendiquait, après sa sortie de la clandestinité, dans le programme d'Erfurt, "l'autodétermination et self-government du peuple" également dans les communes, mais cela ne débouchait pas sur un programme de politique communale cohérent ${ }^{19}$. Le peu d'intérêt du parti pour les questions de politique communale provenait tout d'abord du fait que le parti était exclu de la représentation politique au plan communal par le système électoral en vigueur. Alors que le taux des votes socialistes aux élections du Reichstag était dans de nombreuses villes de beaucoup plus que $50 \%$, un droit de vote censitaire extrême, comme par exemple en Prusse, empêchait une représentation correspondante dans les conseils municipaux ${ }^{20}$. Il s'y ajoutait un rapport traditionnel à l'État qui avait déjà pris des traits idéologiques. L'idée de self-government n'y tenait pas une place importante et, pour cette raison, les principes de politique communale qui furent arrêtés au congrès de Brême en 1904 furent traités du côté libéral de "compromis malheureux de centralisation et de décentralisation". On revendiquait trop peu d'autonomie et de self-government, et trop d'intervention étatique $^{21}$. Le livre de Lindemann gagnait son importance et aussi son caractère brûlant pour le mouvement ouvrier du fait qu'il était en rapport étroit avec les tendances révisionnistes d'Eduard Bernstein. Celui-ci avait exprimé en 1899 sa critique fondamentale de la théorie et de la pratique du $\operatorname{SPD}^{22}$. Sans compter le refus de la théorie catastrophiste de Marx et le renoncement à un but exprimé dogmatiquement, Bernstein s'étendait aussi sur les "tâches prochaines de la social-démocratie". Au nombre de celles-ci, on comptait également la politique communale qui était "depuis longtemps la ou une laissée pour compte du mouvement socialiste",23. Concrètement, il s'agissait "d'utiliser dès aujourd'hui les municipalités comme levier du travail de réforme socialiste et, sans renoncer à

${ }^{18}$ Voir Karl BÜCHER, Die wirtschaftlichen Aufgaben der modernen Stadtgemeinde (1898), dans: ID., Die Entstehung der Volkswirtschaft II, Tübingen ${ }^{7} 1922$, p. 427.

${ }^{19}$ Voir Adelheid VON SALDERN, Sozialdemokratische Kommunalpolitik in Wilhelminischer Zeit, dans: Karl-Heinz NASSMACHER (s.l.d.d.), Kommunalpolitik und Sozialdemokratie, Bonn 1977, p. 18-62; ID., SPD und Kommunalpolitik im Deutschen Kaiserreich, dans: Archiv für Kommunalwissenschaften 23 (1984) II p. 193-214.

${ }^{20}$ Le taux des votes du SPD aux élections du Reichstag 1912 était $49,3 \%$ aux communes audessus de 10000 habitants, alors qu'il était de 34,8\% au total. Voir Helga GREBING, Arbeiterbewegung. Sozialer Protest und kollektive Interessenvertretung bis 1914, München 1985.

21 "Unglücklicher Kompromiß zwischen Zentralisation und Dezentralisation", voir Robert SCHACHNER, Gemeinde und Sozialdemokratie, dans: Archiv für Sozialwissenschaft und Sozialpolitik 23 (1906) p. 763-785, ici 785 .

${ }^{22}$ Eduard BERNSTEIN, Die Voraussetzungen des Sozialismus und die Aufgaben der Sozialdemokratie, 1899, ${ }^{2} 1921$ (réimpr. Reinbek 1969); l'argumentation se trouve déjà dans une série d'articles au "Neue Zeit" parue en 1896-98.

${ }^{23}$ BERNSTEIN, Die Voraussetzungen des Sozialismus 1899, p. 191. 
1'action parlementaire, de s'attaquer à la réalisation des revendications socialistes à partir de la commune" ${ }^{24}$. Ces revendications socialistes communales étaient avant tout la démocratisation du droit de vote, l'extension du droit d'expropriation, aussi bien que la pleine autonomie de la municipalité, particulièrement de la police, par rapport à l'État. Du côté des communes, il fallait, outre la réalisation d'une politique fiscale et scolaire telle qu'elle avait été établie dans le programme de base, maintenant, avant tout, revendiquer l'extension des entreprises municipales et le développement de la politique ouvrière. Pour les établissements municipaux, il fallait poser en principe "que toutes les entreprises à caractère monopoliste et prévues pour les besoins généraux des membres de la commune doivent être exploitées en régie par la commune et que par ailleurs la commune doive tenter d'étendre constamment les limites des services pour ses ressortissants" $"$. Comme employeur, les municipalités devaient jouer un rôle d'exemple non seulement en exploitant des entreprises socialistes modèles, mais aussi sur le marché des travaux publics, où la reconnaissance des conventions collectives et de la liberté de coalition représentaient les revendications minimales. En outre, le SM attendait des villes la création de conseils de prud'hommes et de bourses du travail à caractère paritaire, de même que les communes devaient aussi s'occuper, avec la participation des syndicats, de l'assurance chômage. Tel est à grands traits le SM tel qu'il était formulé dans l'optique révisionniste. Quoique la régie ne fût qu'un des éléments du SM, elle était pourtant perçue comme si importante que les critiques à l'intérieur du parti parlaient pour le dénigrer de "socialisme de l'eau et du gaz". Effectivement Lindemann a donné beaucoup de place au traitement de ce thème dans son ouvrage sur l'administration municipale allemande ${ }^{26}$. En mettant en évidence pour la première fois l'étendue du champ d'action des politiciens communaux socialistes, Bernstein, qui profitait, comme Lindemann, des expériences faites en Angleterre, ne laissait planer aucun doute sur le fait que le SM serait incomplet sans droit de vote démocratique $^{27}$. Le SM était donc l'expression d'une pratique révisionniste. Mais, au contraire de l'exemple fabien, qui avait sans aucun doute exercé son influence

24 "Die Munizipalitäten heute schon als Hebel sozialistischer Reformarbeit zu benützen und, ohne deshalb von der parlamentarischen Aktion abzusehen, von der Gemeinde her an die Verwirklichung sozialistischer Forderungen zu gehen"; ibid.

${ }^{25}$ Ibid., p. 193; "daß alle auf das allgemeine Bedürfnis der Gemeindemitglieder berechneten und Monopolcharakter tragenden Unternehmungen von der Gemeinde in eigener Regie zu betreiben sind und daß im übrigen die Gemeinde danach streben soll, den Kreis der Leistungen für ihre Angehörigen beständig zu erweitern."

${ }^{26}$ Voir Hugo LindemanN, Die deutsche Städteverwaltung. Ihre Aufgaben auf den Gebieten der Volkshygiene, des Städtebaus und des Wohnungswesens, Stuttgart ${ }^{2} 1906\left({ }^{1} 1901\right)$; ID., Arbeiterpolitik und Wirtschaftspflege in der deutschen Städteverwaltung, t. 1.2., Stuttgart 1904; le deuxième tome ("Wirtschaftspflege") est surtout consacré aux usines à gaz et de l'électricité aussi qu'aux tramways.

${ }^{27}$ BERNSTEIN, Die Voraussetzungen des Sozialismus 1899, p. 194: "Stückwerk". 
sur Bernstein ${ }^{28}$, "la question restait [ici] ouverte de savoir s'il pourrait naître du SM un ordre social de nature socialiste" ${ }^{\prime 29}$. Incontestablement, l'activité politique communale du SPD faisait preuve d'un essor évident. En 1913, il y avait des élus socialistes dans 3.500 communes. Leur nombre était passé entre 1907 et 1913 de 5000 à $12000^{30}$. Dans 509 villes, il y avait 2753 socialistes au conseil munici$\mathrm{pa}^{31}$. Le mouvement ouvrier était devenu dans les villes allemandes un facteur politique, dont la force ne s'exprimait pas seulement par le nombre de ses représentants. En premier lieu, ils se réunissaient en groupe pour délibérer en commun sur les propositions et pour arrêter une position cohérente ${ }^{32}$.

L'effet de la théorie SM était cependant beaucoup plus grand dans le camp bourgeois. Alors que la politique sociale de l'État avait reçu de nouvelles impulsions après la chute de Bismarck, elle s'interrompit de nouveau dès le départ de son successeur Caprivi. C'est dans la commune, apparemment loin des débats politiques généraux sur le plan national, que semblait se localiser, pour les réformateurs sociaux, l'endroit approprié pour la réalisation de leur programme. D'autant plus que les villes étaient les points brûlants des problèmes sociaux. Les réformateurs sociaux bourgeois ne participaient guère aux discussions de principe, ils dirigeaient plutôt "toute leur force avec une remarquable synergie vers des solutions pragmatiques qui cependant étaient propres à faire levier pour dissoudre pas à pas l'ensemble du système politique" ". Les organisateurs de ce mouvement de réforme étaient tout d'abord le "Verein für Socialpolitik" et, comme celui-ci prenait de plus en plus le caractère d'une association purement scientifique, la "Gesellschaft für sociale Reform". Fondée en 1901 comme section allemande de l'Association Internationale pour la Protection des Ouvriers ${ }^{34}$, elle réunissait des forces réformatrices bourgeoises qui s'étaient groupées depuis longtemps autour de la revue "Soziale Praxis",35. C'est là que parut en 1897, sous la plume d'Emil Muensterberg, "Stadtrat" berlinois, la première prise de position

${ }^{28}$ Voir Herbert FREl, Fabianismus und Bernstein'scher Revisionismus 1884-1900, Bern, Frankfurt, Las Vegas 1979, p. 90-117.

${ }^{29}$ VON SALDERN, SPD und Kommunalpolitik 1984, p. 203: "konzeptionell offen, wie aus dem Munizipalsozialismus eine Gesellschaftsordnung sozialistischen Zuschnitts hätte entstehen können."

${ }^{30}$ VON SALDERN, Sozialdemokratische Kommunalpolitik 1977, p. 29.

${ }^{31}$ Wolfgang R. KRABBE, Die deutsche Stadt im 19. und 20. Jahrhundert, Göttingen 1989, p. 151.

32 Ibid., p. 150.

${ }^{33}$ Rüdiger VOM BRUCH, Bürgerliche Sozialreform im deutschen Kaiserreich, dans: ID. (s.l.d.d.), "Weder Kommunismus noch Kapitalismus". Bürgerliche Sozialreform in Deutschland vom Vormärz bis zur Ära Adenauer, München 1985, p. 61-179, ici 83: “ihre ganze Kraft mit bemerkenswert gebündelter Energie auf pragmatische Lösungen, die allerdings nicht selten Hebelwirkungen für schrittweise Auflockerungen des politischen Gesamtsystems auszulösen geeignet waren."

${ }^{34}$ Fondée en 1900 à Paris lors de l'exposition universelle.

${ }^{35}$ VOM BRUCH, Bürgerliche Sozialreform 1985, p. 138. 
programmatique sur le SM sans toutefois le désigner comme tel ${ }^{36}$. Tout naturellement aussi des activités d'un caractère commercial privé sont regardées ici comme faisant partie de la politique sociale communale: "Il s'agit ici d'un devoir social de la commune, qu'elle soustraie les entreprises à l'emprise des monopoles dans l'intérêt des plus pauvres ou qu'elle empêche leur livraison aux monopolistes pour les exploiter elle-même pour le bien de la collectivité, ${ }^{37}$. En faisant cela, les entreprises municipales devaient faire principalement attention à la condition des ouvriers et donner ici l'exemple. En outre, elles devaient fournir des services de la meilleure qualité et au meilleur prix possible. Sur le plan théorique, il était difficile d'établir les limites entre régie et entreprise privée, d'autant plus qu'on manquait encore d'expérience. Au plan technique, il n'y a aucune réserve sur l'exploitation par la municipalité des services et des transports. Alors qu'en Angleterre on est allé très loin avec la municipalisation, en Allemagne ne peuvent être considérés comme entre les mains des communes que l'approvisionnement en eau et le service des ordures. En ce qui concerne l'éclairage, Muensterberg se montrait en revanche beaucoup plus réticent, ce qui s'explique de la part d'un membre de l'administration municipale berlinoise qui était plutôt hostile à la régie. Le SM est considéré ici comme une politique sociale sur le plan communal, dans laquelle les entreprises municipales ont avant tout la fonction d'entreprises modèles sur le plan social qui doivent foumir leurs services au meilleur prix possible.

Pour un membre éminent de la jeune école historique de l'économie politique allemande, les entreprises municipales ont en revanche une importance beaucoup plus grande. Dès 1898, le professeur Karl Bücher de Leipzig comptait parmi les plus importants devoirs économiques de la grande ville moderne "la création d'établissements d'utilité publique" 38 . Outre les arguments de politique sociale déjà connus prend place ici en premier lieu l'intérêt fiscal. Des entreprises municipales fourniraient des rentrées qui devraient être réunies autrement par la voie fiscale $^{39}$. Le monopole naturel joue également la plupart du temps en faveur de la régie. Celle-ci en outre n'est en aucune façon inférieure aux sociétés anonymes privées, car les deux doivent travailler avec des employés: "Je ne vois pas pourquoi les employés privés seraient plus économes, plus efficaces que les fonctionnaires publics soumis à un contrôle permanent" ${ }^{\circ 0}$. Les municipalités fonderaient et exploiteraient même ces établissements moins cher, car elles utilisent des effets de synergie et réalisent des économies d'échelle.

\footnotetext{
${ }^{36}$ Kommunale Sozialpolitik, dans: Soziale Praxis 7 (1897-98) p. 161-166.

${ }^{37}$ Ibid. p. 163: "Hier handelt es sich um eine soziale Aufgabe der Gemeinden dann, wenn sie die Unternehmungen aus ihrer eng begrenzten Monopolstellung weniger Bevorzugter herausnimmt oder ihre Auslieferung an Monopolisten verhütet und die Unternehmungen zum Besten der Gemeinschaft selbst betreibt."

${ }^{38}$ BÜCHER (cf. note 18) p. 425-430; "Errichtung gemeinwirtschaftlicher Anstalten".

${ }^{39}$ Ibid., p. 427: "der ja immer für die Bevölkerung etwas Schmerzliches hat."

${ }^{40}$ Ibid., p. 426: "Weshalb Privatbeamte sparsamer, wirtschaftlicher verfahren sollen als die einer fortgesetzten Kontrolle unterworfenen öffentlichen Beamten, ist schwer einzusehen."
} 
Un autre protagoniste éminent du socialisme de la chaire se prononçait déjà en faveur d'un financement de la politique sociale communale par l'extension de la régie $^{41}$. De telles opinions se répandaient rapidement. La municipalisation des services monopolistes fut déclarée un devoir éminent de politique sociale ${ }^{42}$. Les bénéfices s'y réalisant en toute sécurité devaient revenir à la municipalité pour permettre de supprimer des impôts antisociaux comme par exemple l'octroi ${ }^{43}$.

Au premier congrès des villes allemandes en 1903, le SM, "GemeindeSozialismus" ou "Kommunal-Sozialismus", fut élevé au rang de thème central. Jusque dans les milieux bourgeois, ces idées recevaient un tel accueil ${ }^{44}$ que le premier bourgmestre de Francfort Adickes se voyait obligé de faire une mise au point: il faut nettement distinguer le SM comme nouvelle tendance politique, "mettant en rapport depuis peu le socialisme avec l'administration des villes", de la politique sociale comme activité réformatrice de l'État et des municipalités ${ }^{45}$. C'est seulement ainsi que l'on pourrait juger les revendications des programmes socialistes à l'épreuve de leur viabilité, sans préjugés. Il ne contestait pas qu'il en découlait "des progrès sains et remarquables dans le sens de la modernisation", mais il fallait peser exactement leur portée et leur importance fondamentale. Il est urgent d'établir un "programme social municipal" bourgeois pour l'opposer aux revendications socialistes ${ }^{46}$. Dans un tel programme se trouvent bien entendu des entreprises municipales à caractère monopoliste. Il faut continuer avec "prudence et conséquence" le "processus du municipalisation" déjà en voie depuis assez longtemps, bien que le fonctionnaire expérimenté ne sous-estime pas les problèmes de la régie ${ }^{47}$. En tout cas, il faut réaliser des bénéfices servant à la couverture du risque commercial, mais également au remboursement des déficits des autres ressorts. Son collègue de Dresde Beutler parlait beaucoup plus franchement en faveur de la régie. Certes, on ne peut pas déterminer théoriquement l'étendue de la régie, c'est une question purement pratique, avec à la rigueur des considérations sociales secondaires. Sans ambages, le premier bourgmestre de Dresde évoquait le fait qu'on peut "gagner un bon paquet d'argent" par exemple avec le gaz et l'électricité ${ }^{48}$. En conséquence il faut gérer ces établissements comme des

\footnotetext{
${ }^{41}$ Heinrich HERKNER, Die Arbeiterfrage. Eine Einführung, Berlin ${ }^{2} 1897$, p. 406-440, ici 436sv.

${ }^{42}$ Karl TRIMBORN, Otto THISSEN, Die Thätigkeit der Gemeinden auf socialem Gebiete (Communale Socialpolitik), Mönchen-Gladbach 1900 (Sociale Tagesfragen, 8.-11. Heft).

${ }^{43}$ Adolf DAMASCHKE, Vom Gemeinde-Sozialismus, 8. Tausend, Berlin s.d. [1901] (Soziale Streitfragen, 1) p. 132-140.

${ }^{44}$ Voir par exemple le compte rendu très positif de l'ouvrage de LINDEMANN, Die deutsche Städteverwaltung, Stuttgart 1901, par Hjalmar SCHACHT dans les Preußische Jahrbücher 105 (1901) p. 352-354.

${ }^{45}$ Voir ADICKES, BEUTLER, Die sozialen Aufgaben der deutschen Städte. Zwei Vorttäge gehalten auf dem ersten deutschen Städtetage zu Dresden am 2. September 1903, Leipzig 1903, p. 11, 26: "welche neuerdings den Sozialismus mit der Stadtverwaltung in enge Beziehung gesetzt hat".

${ }^{46}$ Ibid., p. 68.

${ }^{47}$ Ibid., p. 29.

${ }^{48}$ Ibid., p. 123.
} 
entreprises privées. On se servirait des bénéfices "appropriés" pour alléger la charge fiscale. Il est évident que ce point de fiscaliste fut soumis à d'ardentes critiques dans le mouvement ouvrier. "Car outre la possibilité de renoncer au profit, c'est la tarification sociale qui est la principale justification de la régie"49.

Le développement rapide des entreprises municipales et de la politique sociale communale attira de bonne heure l'attention des socialistes de la chaire. Dès 1901, une enquête sur ce thème avait été suggérée dans le "Verein für Socialpolitik". Gustav Schmoller lui-même s'occupa de la question. En 1903, un programme de travail pour l'enquête sur les communes fut présenté ${ }^{50}$. L'étude qui n'était pas limitée à la situation en Allemagne incluait aussi, entre autres, l'Autriche, la France et l'Angleterre, déboucha sur la plus grande publication du Verein jusqu'à présent ${ }^{5 !}$. Avec la discussion du sujet aux deux conférences du Verein en 1907 à Magdebourg et en 1909 à Vienne, le débat sur le SM atteignait en Allemagne son apogée. Il est vrai que la question la plus importante dans les débats était la législation électorale des communes, mais l'opinion que les communes avaient à remplir des devoirs de politique sociale s'était déjà vulgarisée parmi tous les penseurs. On refusait naturellement le "municipalisme social des sociaux-démocrates, qui tendait à conquérir d'abord les communes pour conquérir ensuite l'État ${ }^{, 52}$. Le praticien expérimenté de l'administration municipale n'avait cependant aucune phobie des contacts avec le SM. Il faut que la ville prenne en mains les affaires qui ont un caractère monopoliste ${ }^{53}$. Dans le débat se faisait entendre une critique de cette définition du SM: une telle sorte de municipalisation n'a rien à voir avec la politique sociale ou le socialisme, mais au contraire est "plutôt du pur fiscalisme"

L'adepte éminent du socialisme d'État, Adolph Wagner, plaidant pour la muni-

49 "Denn außer in der Möglichkeit des Verzichts auf Profit liegt in der sozialpolitischen Gestaltung der Tarife der Hauptrechtfertigungsgrund städtischer Regie."; "Die Finanznot der Städte ist stets größer geworden, und da man vor einer tiefgreifenden Besteuerung der Einkommen, Vermögen und Erbschaften und ebensosehr der Konjunkturengewinne zurückschreckt, dieselbe auch bei den heutigen politischen Machtverhältnissen undurchführbar ist, so greift man zur Munizipalisierung monopolistischer Privatunternehmungen und zur Ausbeutung derselben nach privatkapitalistischen Grundsätzen." LINDEMANN, Arbeiterpolitik und Wirtschaftspflege 1901, t. 2, p. 84 .

${ }^{50}$ Voir BOESE, Geschichte des Vereins für Sozialpolitik 1939, p. 98.

${ }^{51}$ Elle comprend 6323 pages des Schriften des Vereins für Sozialpolitik, se partageant entre les volumes 117-123 (Verfassung und Verwaltungsorganisation der Städte), 126, 127 (Gemeindefinanzen) et 128-130 (Gemeindebetriebe. Neuere Versuche und Erfahrungen über die Ausdehnung der kommunalen Tätigkeit in Deutschland und im Ausland).

52 "Sozialdemokratischer Sozialmunizipalismus, der zunächst die Gemeinden erobern will, um dann den Staat zu erobern."; ainsi le rapporteur le professeur Edgar Loening à Magdebourg en 1907; voir Verhandlungen der Generalversammlung in Magdeburg 1907, Leipzig 1908 (SVfSP, 125) p. 173.

${ }^{53}$ Voir les remarques de Karl Flesch, "Stadtrat" de Francfort: "gewisse Dinge in öffentlichen Betrieb nehmen, insofern also M. zu treiben." Ibid., p. 227, 230.

${ }^{54}$ Voir les remarques de "Stadtrat" Voßberg de Berlin-Schöneberg; ibid., p. 290. 
cipalisation des entreprises monopolistes, se référait ainsi aux revendications qu'il avait soutenues dès 1873: sous l'impression immédiate du "Krach", il avait demandé la prise de contrôle des secteurs du transport et de l'approvisionnement par des entreprises publiques ${ }^{55}$. En 1909, à Vienne, il constatait avec satisfaction un consensus sur ses vues ${ }^{56}$. La municipalisation fut unanimement reconnue comme un "progrès culturel en tout" ". On n'appréhendait pas d'appeler SM l'érection et l'extension des entreprises municipales, bien que cela n'eût rien à faire avec le socialisme au sens propre ${ }^{58}$. En regard de ce consensus, la discussion de la question de l'extension que devrait avoir cette municipalisation a tourné court. Alors que la régie, dans le domaine des services était considérée comme éprouvée et n'était plus discutée, elle n'était pas encore généralement acceptée dans les transports. Dans d'autres domaines (ravitaillement, logement), elle n'était la plupart du temps qu'au stade des essais ${ }^{59}$. La question resta également contestée de savoir si et dans quelle mesure les entreprises municipales devaient réaliser des bénéfices. On opposait le reproche de "fiscalisme municipal" aux vastes programmes des hauts fonctionnaires municipaux ${ }^{60}$. Même les frères Alfred et Max Weber ne parvenaient pas à ébranler le consensus général d'après lequel il fallait au moins municipaliser les entreprises monopolistes qui utilisaient la voirie. Dans une discussion en grande partie technique, ils rencontraient peu d'approbation de leur critique fondamentale des tendances à la bureaucratisation liées à l'extension progressive des entreprises municipales. Leur appel à détruire la "métaphysique de la bureaucratie" restait sans écho, tout comme les réserves formulées par Mombert à l'encontre de Wagner, selon lesquelles une municipalisation trop étendue aurait des conséquences négatives pour la productivité et par conséquent aussi pour la distribution ${ }^{62}$.

L'étude du SM dans le "Verein" marque un certain tournant. D'un côté se montraient déjà les débuts d'un nouveau développement technique et organisationnel des entreprises municipales. Les zones de production franchissaient les limites communales et des capitaux publics et privés se réunissaient en grandes entreprises d'économie mixte. D'autre part la critique du SM se renforçait ${ }^{63}$. Le

${ }^{55}$ Voir la communication de Wagner sur la question des sociétés anonymes à la deuxième conférence d'Eisenach (SVfSP, 4) p. 52.

${ }^{56}$ Voir Verhandlungen des Vereins für Socialpolitik in Wien 1909, Leipzig 1910 (SVfSP, 132) p. $241,253$.

${ }^{57}$ Ibid., p. 322: "Kulturfortschritt im ganzen".

${ }^{58}$ Voir le rapporteur FUCHS, Ibid., p. 132: "das Wort "Sozialismus" schreckt uns hier in diesem

Kreise nicht, aber es begeistert uns auch nicht."

${ }^{59}$ Voir Carl Johannes FUCHS, Vorwort, dans: ID. (s.l.d.d.), Gemeindebetriebe, vol. I, Leipzig 1908 (SVfSP, 128) p. IX.

${ }^{60}$ Ibid., p. 229, 318.

${ }^{61}$ Ibid., p. 312; voir aussi BOESE, Geschichte des Vereins für Sozialpolitik 1939, p. 133.

${ }^{62}$ Ibid., p. I69, 318.

${ }^{63}$ Pour le moment encore très mesuré dans le Zeitschrift für Sozialwissenschaft, édité par Julius Wolf, opposant éminent du socialisme de la chaire; voir Georg JAFFÉ, Die wirtschaftlichen 
socialisme de la chaire perdait sa prédominance idéologique, politique et science divergeaient maintenant irréversiblement ${ }^{64}$. Le célèbre écrit polémique de Lord Avebury contre le SM anglais fut publié en Allemagne par un adepte éminent de la science économique exacte ${ }^{65}$. Une protestation publique se fit aussi entendre en Allemagne, mais sans atteindre la même intensité qu'en Angleterre ${ }^{66}$. Se concentrant de plus en plus sur la question de leur productivité comparée à celle des entreprises privées, le débat sur les entreprises communales perdait son principal caractère. Les socialistes de la chaire hésitaient à s'engager dans cette discussion. Elle se déplaçait vers les cercles d'experts ${ }^{67}$, le débat se concentrant particulièrement sur les usines électriques, car ici il y avait non seulement des entreprises municipales, mais aussi des entreprises privées en grand nombre, ce qui permettait une comparaison directe. Cette importance particulière attachée aux usines électriques nous amène à nous interroger sur leur place dans le développement du SM.

\section{La municipalisation de l'électricité}

Jetons d'abord un coup d'œil sur le mouvement quantitatif entre 1890 et $1913^{68}$.

Unternehmungen der Städte, vol. 11 (1908), p. 414-437; il mettait en garde contre une extension continue des entreprises municipales anticipant les doutes des frères Weber: "Wer nicht blinder Anhänger des staatlichen oder städtischen Sozialismus ist, kann nicht wünschen, daß immer größere Mengen von Bürgern den freien Berufen entzogen und von einer leitenden Stelle abhängig werden." p. 436.

${ }^{64}$ VOM BRUCH, Bürgerliche Sozialreform 1985, p. 125.

${ }^{65}$ Voir Lord AVEBURY (i.e. John Lubbock), Staat und Stadt als Betriebsunternehmer, Berlin 1909. Richard Ehrenberg, plongé dans une polémique ardent avec les socialistes de la chaire l'année prochaine, écrivit l'avant-propos; voir Hans GEHRIG, Die Begründung des Prinzips der Sozialreform, Jena 1914 , p. 348-353.

${ }^{66}$ L'éminent journal économique "Der deutsche Ökonomist" mettait en garde contre une évolution "welche darauf hinausläuft, den Spielraum für private Unternehmertätigkeit immer mehr einzuengen, um sie allmählich ganz auszuschalten und somit unsere Volkswirtschaft auf völlig sozialistische und kommunistische Grundlage zu überführen." Voir l'article "Gemeinde- und Staatssozialismus", 1909, p. 633sv.; un peu plus moderé encore en 1906, p. 572. Le "Handwerksund Gewerbekammertag", organisation des petits artisans critiquait en 1909 l'extension progressive de la régie; voir Kommunales Jahrbuch 3 (1910) p. 520.

${ }^{67}$ Voir LEONI, Die Verbindung von Gemeinden und Privatkapital zu wirtschaftlichen Unternehmungen, dans: Technik und Wirtschaft 7 (1914) p. 532-558, 835-837; il s'agit d'une communication du maire adjoint de Strasbourg présentée au "Deutscher Städtetag"; voir Verhandlungen des 4. Deutschen Städtetages am 15. und 16. Juni 1914 zu Cöln, Berlin 1914, p. 4675 avec la discussion et les directives votées mais sans l'appendice statistique. Voir aussi l'examen d'Avebury chez Emil SCHIFF, Staat und Stadt als Betriebsunternehmer, dans: Technik und Wirtschaft 2 (1909) p. 433-446, 481-496, 529-541.

${ }^{68}$ Voir diagrammes, p. 95. 
En ce qui concerne le nombre des usines, le taux de la propriété publique, c.-à-d. normalement communale, a diminué d'un tiers à plus d'un quart. Pour la capacité de production par contre, ce taux est monté de $31 \%$ à plus de $37 \%$. En 1911, il a même été de $40 \%$. Ces chiffres globaux ne suffisent pas pour une étude de la municipalisation. Il faut plutôt regarder le développement dans les villes, plus précisément dans les grandes villes. Il n'existe pas encore de statistique complète, mais nous pouvons recourir aux études déjà mentionnées du "Verein" celles-ci, toutes les villes de plus de 50000 habitants avaient un service public d'électricité en 1907, dont les trois quarts étaient en propriété communale. Dans les grandes villes au sens propre ${ }^{70}$, le taux de la régie montait même à $80 \%$. Par la suite, ce chiffre tomba un peu parce que le nombre des entreprises d'économie mixte s'est accru. Mais en 1913 encore, il n'y avait que six usines électriques privées dans les 47 grandes villes ${ }^{71}$. Cette quote-part élevée des régies vient tout d'abord de ce que les usines de loin les plus nombreuses étaient déjà fondées comme entreprises municipales, à savoir 33 au total. Les douze fondations privées se réduisaient de moitié par municipalisation ou transformation en entreprise d'économie mixte.

Comment faut-il expliquer ce résultat? Evidemment, les villes ne reculaient pas devant la perspective de fonder elles-mêmes leurs usines électriques, un fait d'autant plus remarquable qu'il s'agissait d'une technique nouvelle et certainement pas complètement mûrie jusque dans les années 1890 . Quelles raisons peuton trouver à ce goût du risque? A l'inverse, qu'est-ce qui inclinait néanmoins quelques villes à une solution d'économie privée ${ }^{72}$ ? Il faut pour cela examiner à titre d'exemple le développement dans les villes prises individuellement. Nous mettons les pionniers au premier plan, car ces fondations précoces servirent sans doute de modèle.

Au début, il y a Berlin, et il est tout à fait remarquable que le modèle de la capitale n'ait pas fait école. Peu après sa fondation, la Deutsche Edison-Gesellschaft s'adressa à la ville de Berlin pour demander une concession pour une centrale électrique. La municipalité ne voulait pas se refuser à ce développement, d'autant plus que les installations privées, qui poussaient comme des champignons, concurrençaient l'usine à gaz municipale, sans que la ville puisse obtenir une compensation financière. L'exploitation d'une telle entreprise en régie, comme le revendiquaient les conseillers socialistes, fut refusée "pour le moment". Comme motifs, on invoquait avant tout le risque financier, sans compter le manque de compétence technique et commerciale, car, vu qu'il s'agissait d'un

\footnotetext{
${ }^{69}$ Voir Paul MOMBERT, Die Gemeindebetriebe in Deutschland. Allgemeine Darstellung, dans: (SVfSP, 128, I) p. 1-77, notamment p. 54-73.

${ }^{70}$ Villes avec plus de 100000 habitants.

${ }^{71}$ Il s'agit des grandes villes sur la base du recensement de la population de 1910: Augsbourg, Berlin, Berlin-Schöneberg, Brunswick, Plauen et Stettin; il y avait des entreprises d'économie mixte à Altona, Essen, Hambourg et Strasbourg; Königsberg était affermé à l'AEG.

${ }^{72}$ On peut $y$ ajouter les entreprises en affermage.
} 


\section{Leistung}

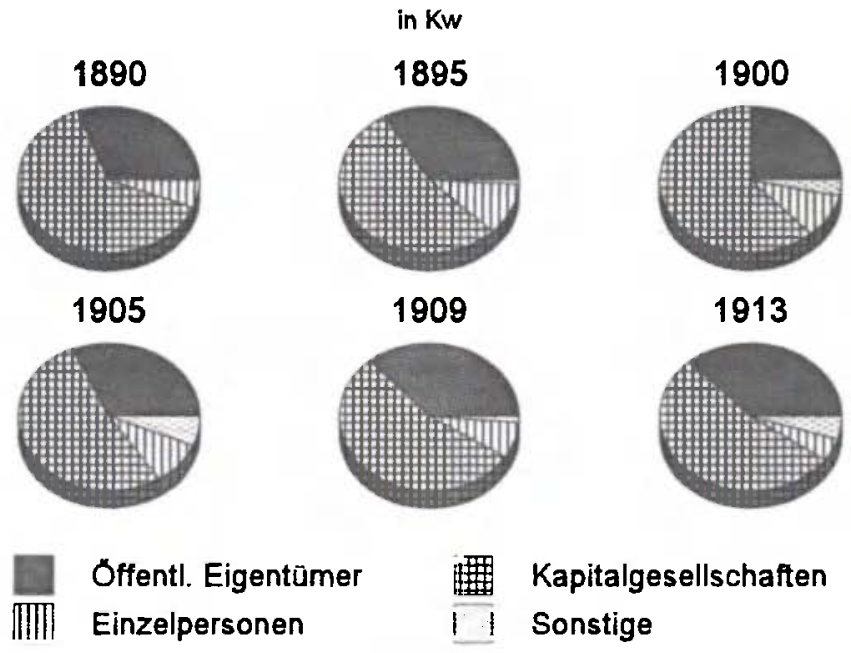

Installierte Leistung nach Eigentumsverhältnissen, Deutsches Reich 1890-1913.

\section{Anzahl}
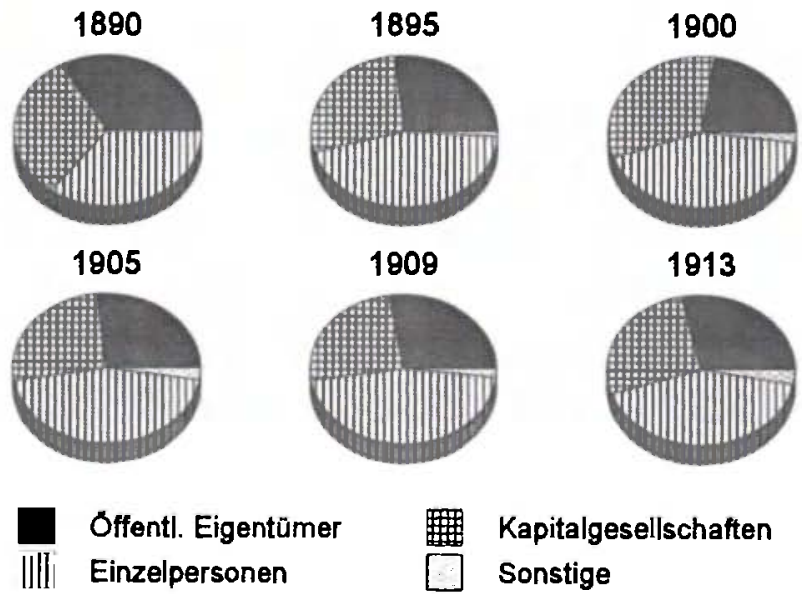

Anzahl nach Eigentumsverhältnissen, Deutsches Reich 1890-1913.

Quelle: Hugo OrT (Hg.), Statistik der öffentlichen Elektrizitätsversorgung Deutschlands 1890-1913, St. Katharinen 1986, S. XXXIII f. 
"commerce très hasardeux", il n'était pas justifié d'y employer "la fortune et les impôts de la collectivité" ${ }^{73}$. En cas de régie, on ne pourrait pas se limiter à l'alimentation du centre, ce qui nécessiterait d'énormes moyens financiers. Le contrat de concession conclu avec la DEG en 1884 pour l'utilisation d'une certaine partie de la voirie dans le centre ville était cependant si peu précis que très vite les partenaires entrèrent en conflit. Pour une autre extension du service, la ville dut payer les Berliner Elektrizitäts-Werke (BEW) par d'importantes concessions. Certes, le nombre des partisans d'une municipalisation augmenta par la suite mais ils ne purent pas imposer leur volonté à cause du caractère ploutocratique de la législation électorale quand, en 1898 , il y eut un nouveau contrat à voter $^{74}$. Ce n'est qu'en 1915 que les BEW furent transformées en entreprise d'économie mixte.

Les villes allemandes dans lesquelles furent fondées par la suite des centrales électriques s'engagèrent dans un chemin différent. La respectable ville hanséatique de Lübeck décida en 1886 de fonder une usine municipale. L'accent étant mis sur les avantages hygiéniques du nouvel éclairage, on ne pouvait en aucun cas laisser ce service à un monopole privé ${ }^{75}$. La décision des villes d'Elberfeld (1887) et Barmen (1888) n'avait rien à voir avec des arguments idéologiques. Il s'agissait ici d'une municipalisation envisagée dès le début uniquement pour sauvegarder les bénéfices des usines à gaz municipales et le cas échéant également les profits des services d'électricité. Tout cela servait à un allègement des impôts et n'avait rien à voir avec un SM motivé par des objectifs de politique sociale ${ }^{76}$. A Magdebourg également, le choix de la régie avait été fait dès 1887 parce que c'était le seul moyen d'empêcher une concurrence dangereuse pour l'usine à gaz municipale. En cas de concession il aurait fallu poser des conditions tellement léonines qu'aucun entrepreneur privé n'aurait été disposé à les accepter. La controverse dite "Systemstreit" empêcha la mise en pratique de la décision. Vu que l'électricité n'était absolument pas une concurrence destructrice pour le gaz, on opta en 1895 pour une concession à l'AEG. Si la ville fut dans l'immédiat très satisfaite, peu après par contre il survint un différend sur le partage des bénéfices. Pour cette raison,

${ }^{73}$ Ainsi le premier bourgmestre Forckenbeck aux débats du conseil municipal; cité par Wolfram FISCHER, Die Elektrizitätswirtschaft in Gesellschaft und Politik, dans: ID.(s.l.d.d.), Geschichte der Stromversorgung, Frankfurt a. M. 1992, p. 15-36, 25. Voir aussi le récit chez LINDEMANN, Arbeiterpolitik und Wirtschaftspflege 1901, t. 2, p. 115-125.

${ }^{74}$ Ibid., p. 124.

${ }^{75}$ Voir Uwe KÜHL, Die Gründung des ersten kommunalen Elektrizitätswerks Deutschlands in Lübeck, dans: Zeitschrift des Vereins für Lübeckische Geschichte und Altertumskunde 79 (1999), p. 237-271.

${ }^{76}$ Voir Fritz Dieter ERBSLOH, Die frühen elektrischen Zentralanlagen in den Nachbarstädten Elberfeld und Barmen, Frankfurt a. M. 1995, p. 304-309.

${ }^{77}$ Sur les avantages et les inconvénients du courant continu et du courant alternatif voir Wolfgang KÖNiG, Massenproduktion und Technikkonsum, dans: ID. (s.l.d.d.), Propyläen-Technikgeschichte, t. 4,1990, p. 331-335. 
l'entreprise fut municipalisée le plus tôt possible après dix ans d'exploitation ${ }^{78}$. On assiste au même déroulement des événements à Leipzig où l'on croyait atteindre le but plus vite avec un concessionnaire pour prévenir l'implantation de plus grandes installations privées. Les conflits avec le concessionnaire s'amorcèrent sur la question de l'extension du service, que l'entreprise appartenant au groupe Siemens \& Halske n'était prête à accepter qu'après l'autorisation d'une augmentation des tarifs. Ici aussi, la municipalisation survint au bout de dix ans, comme le permettait le contrat ${ }^{79}$. A Königsberg, l'insuffisance des offres des entrepreneurs poussa la ville en 1890 à mettre en régie l'entreprise dans le but de réaliser des bénéfices pour le budget de la ville ${ }^{80}$. L'appréhension qu'une entreprise privée ne se limitât à l'alimentation lucrative d'un rayon très limité fut également à Brême la vraie raison du choix de la régie ${ }^{81}$. On pourrait multiplier ces exemples à volonté.

\section{Conclusion}

Il est donc clair que des considérations fiscales étaient au premier rang des raisons de la municipalisation des usines électriques, pour l'instant d'une manière plutôt défensive pour repousser une nouvelle concurrence. Il s'agissait de sauvegarder les rentrées de sa propre usine à gaz. De prime abord on ne pouvait pas encore prévoir que la nouvelle forme d'énergie aurait peu de conséquences négatives pour le gaz. Comme il devint sensible qu'ils se complétaient au contraire, les bénéfices à attendre de la fourniture de courant furent un argument de plus pour sa municipalisation. Pour les avocats du vrai SM, ce développement se présentait comme un pur fiscalisme: "Les municipalités prenant l'exploitation des usines à gaz, électriques, etc. en régie ont conservé les méthodes de l'économie privée capitaliste et elles se sont laissé guider par le principe fiscal du profit maximal. Elles ont continué à exploiter les entreprises privées en tant que telles au lieu de

${ }^{78}$ Voir Otto LANDSBERG, Die Betriebe der Stadt Magdeburg, dans: C. J. FUCHS (s.l.d.d.), Die Gemeindebetriebe der Städte Magdeburg, Naumburg a. S. und Frankfurt a. M., Leipzig 1909 (SVfSP, 129, 3) p. 58-62; LINDEMANN, Arbeiterpolitik und Wirtschaftspflege 1901, t. 2, p. 113 sv.

${ }_{79}^{79}$ Voir Paul WeIGEL, Die Gemeindebetriebe der Stadt Leipzig, Leipzig 1909 (SVfSP, 129,7) p. 35-39.

${ }^{80}$ Voir Georg NeuHaus, Die Gemeindebetriebe der Stadt Königsberg i. Pr., Leipzig 1910 (SVfSP, 129,9) p. 85-101.

${ }^{81}$ Les avocats de la régie craignaient qu'un concessionaire s'abstiendrait d'une extension des installations en cas de perspective incertaine de bénéfice, "während derartige Fragen bei Senat und Bürgerschaft stets eine gerechtere [...] Würdigung erwarten dürfen". Voir Achim SAUR, Eine "Elektrische Centralanlage" für Bremen, dans: Bremen wird hell: hundert Jahre Leben und Arbeiten mit Elektrizităt, Bremen 1993, p. 18-33, ici 27. 
les changer en établissements de l'administration publique et de les gérer suivant les principes qui y président. De telle sorte que n'ont changé que les propriétaires - pour le reste, tout est resté comme par le passé,"82. Bien que la réalisation de rentrées fût le principal mobile de la municipalisation des usines électriques, d'autres considérations jouèrent un rôle, différent de place en place et au cours du temps, lors de la décision pris à l'encontre de l'entreprise privée. Elles reflétaient couramment les expériences négatives avec les compagnies à gaz privées, par exemple la crainte que les extensions nécessaires du réseau ne s'effectuent pas du tout ou seulement insuffisamment. Cela pouvait d'un côté gêner l'urbanisme systématique, d'autre part provoquer des conflits parmi les citoyens, leur désir d'utiliser de nouvelles techniques n'étant pas satisfait. Avec la régie, on pouvait utiliser plus aisément la tarification comme instrument d'une politique communale d'aide économique ${ }^{83}$. L'argument d'un soutien à la classe moyenne jouait ici un rôle particulièrement important ${ }^{84}$. La plupart des politiciens communaux en Allemagne étaient convaincus de ne pas pouvoir atteindre ces divers buts par des contrats de concession. Adolph Wagner doutait également qu'il fût possible "de faire des contrats et de formuler les cahiers des charges de telle manière que les intérêts collectifs soient suffisamment défendus de façon durable, ${ }^{95}$.

La municipalisation des usines électriques était-elle donc dépourvue d'une quelconque importance pour le SM envisagé comme une politique sociale de la commune? Naturellement non, car, suivant l'opinion dominante des contemporains, les entreprises municipales formaient avec leurs bénéfices la base financière qui permettrait par la suite de remplir des devoirs de politique sociale au sens le plus large ${ }^{86}$. Ces détails dépendaient de décisions politiques. Il ne résultait pas nécessairement du fait qu'une entreprise était en régie municipale une rémunération plus élevée des ouvriers qui y étaient employés ${ }^{87}$. Mais on ne fait pas fausse route en supposant que les bénéfices réalisés par les entreprises municipales étaient utilisés absolument dans l'intérêt de la plus grande partie des habitants.

${ }^{82}$ LINDEMANN, Arbeiterpolitik und Wirtschaftspflege 1901, t. 2, p. $106 \mathrm{sv}$.

${ }^{83}$ Voir V. BLUME, article "Gemeinden (sozialpolitische Aufgaben)", dans: Handwörterbuch der Staatswissenschaften, t. 4, Jena ${ }^{3} 1909$, p. 618-628; Karl Christian SCHAEFER, Der Beginn der kommunalen Energieversorgung in Krefeld, dans: Blätter für deutsche Landesgeschichte 127 (1991), p. 271-304, ici 299.

84 "Wichtig ist ferner für viele Kleinbetriebe die Bereitstellung von Naturkraft (Gas, Wasser, Elektrizität) durch die Gemeinden, die schon aus diesem Grunde dahin streben müssen, Herr der kraftliefernden Werke zu werden." voir BLUME, Gemeinden ${ }^{3} 1909$, p. 621.

${ }^{85}$ Verhandlungen der Generalversammlung Magdeburg 1907, p. 283: "Verträge zu schließen und die Lastenhefte so einzurichten, daß dauernd darin die allgemeinen Interessen genügend wahrgenommen werden".

${ }^{86}$ Voir Tae-Yel KWACK, Die Entwicklung von Kommunalunternehmen in Deutschland im 19. und frühen 20. Jahrhundert - unter besonderer Berïcksichtigung finanz- und sozialpolitischer Aspekte, Münster 1990, p. 389; Max WEISS, Die finanzpolitische Bedeutung der Gemeindeunternehmungen. Mündliches Referat, dans: Verhandlungen 1910 (SVfSP, 132) p. 186.

${ }^{87}$ Paul MOMBERT, Schlußwort, dans: Verhandlungen 1910 (SVfSP, 132) p. 315. 
Dès lors, le SM ainsi compris contribuait aussi à la légitimation du système politique en vigueur comme on l'avait invoqué comme raison ${ }^{88}$.

\section{Deutsche Zusammenfassung}

Schon die Zeitgenossen vor dem Ersten Weltkrieg zählten die deutschen Städte zu den am besten verwalteten. Diese Einschätzung beruhte neben anderen Gründen vor allem auf dem großen Umfang und dem breiten Wirkungskreis der Gemeindebetriebe. Gemeindebetriebe bildeten den Kern des Munizipal-Sozialismus (MS) in Deutschland, den man als Korrelat zum StaatsSozialismus ansehen kann. Der Beitrag behandelt zunächst die rasche und weitgehende Rezeption des Begriffs MS, die dem Wirken der sog. "Kathedersozialisten", organisiert im Verein für Socialpolitik, zugeschrieben wird. Im zweiten Teil wird nach den Gründen gefragt, weshalb die deutschen Großstädte von Anbeginn die Elektrizitätsversorgung in Gemeindehand betrieben. Waren es wirklich Ideen eines MS oder gab es vielmehr andere Gründe? Neuere kliometrische Studien zum kommunalen Investitionsverhalten bieten drei Hypothesen für die Bestimmungsgründe an: Investitionen als antizipatorische Steuerminderungsstrategie; Investionen zur Befriedigung einer effektiven Nachfrage einer neuen Dienstleistung; Investitionen als integraler Bestandteil einer sozialpolitischen Strategie. Die Wahl der Eigentumsform läßt sich entweder ideologisch, d.h. durch den MS erklären oder war durch das finanzielle Interesse der Kommune an der Schaffung neuer Einnahmequellen begründet, womit sich dann die Erhöhung direkter Steuern vermeiden ließ. Von den untersuchten 47 deutschen Großstädten besaßen nur sechs ein privates E-Werk, was in erster Linie darauf zurückzuführen ist, $\mathrm{da} B$ diese Betriebe bereits als kommunale gegründet wurden. Dafür waren eindeutig fiskalische Gründe entscheidend, die zunächst einen eher defensiven Charakter hatten, um die Einnahmen aus den Gaswerken, die zumeist in kommunaler Hand waren, zu sichern. Später dienten die zu erwartenden Einnahmen aus der Stromversorgung als zusătzliches Argument für die Kommunalisierung. Für die Vertreter eines wirklichen MS war das purer Fiskalismus: Nur der Eigentümer war ein anderer, nicht aber die Methoden der nach Profitmaximierung strebenden kapitalistischen Privatökonomie. Dieser Vorwurf focht die pragmatisch denkenden und handelnden Bürgermeister nicht an. Gemeindebetriebe stellten sich für sie als integraler Bestandteil einer guten Verwaltung dar, und wurden, um einen Kathedersozialisten zu zitieren, gar als Kulturfortschritt angesehen. Als der Verein für Socialpolitik sich dem Phänomen der Gemeindebetriebe mit einer großen Untersuchung widmete, hatten sich diese bereits weitest möglich verbreitet. Sicher schufen die Kathedersozialisten ein dem Interventionsstaat günstiges ideologische Klima, der MS nach der deutscher Art entstand in den Städten selbst und wurde durch den Zwang der Verhältnisse ausgelöst.

\section{English Abstract}

Already the contemporaries counted german cities before World War I among the best administrated ones. This was beside other reasons highly due to the large scale and wide scope of municipal enterprise in this country. Actually municipal enterprise was the centre-piece of municipal socialism in Germany, which is regarded as correlate of state socialism. This article treats first the very quick and extensive reception of the notion municipal socialism in Germany,

${ }^{88}$ Les masses de l'été 1914 ne partaient pour la guerre seulement pour le roi et la patrie mais aussi pour les villes "qui sont devenues un peu les leurs." Pierre AYÇOBERRY, Les luttes pour le pouvoir dans les grandes villes de l'Allemagne impériale, dans: Le mouvement social 136 (1986) p. 83-102, ici 102. 
which is attributed to the work of the so-called "Kathedersozialisten", this well-known group of economists and social scientists which got together in the "Verein für Socialpolitik" (Society for Social Policy). In a second part, reasons are investigated why the German big cities from the very beginning ran their electric power stations as municipal ones. Did there really exist any ideas of a municipal socialism or were there other considerations? Recent cliometric studies on municipal investment offer three hypotheses for the determinants: first investment as an anticipate strategy of tax relief, second as a response to an effective demand for a certain new service, and finally municipal investment can be interpreted as a social political strategy. As seen in the article, the decision on the form of the property of an urban electricity supply can be explained either by a social political idea, i.e. the municipal socialism, or it was motivated by financial interest of the municipality of creating new sources of revenues and so make possible to do without increasing direct taxes. Examinating the 47 german big cities (with more than 100000 inhabitants) we find among these only 6 with a privately owned power station. This is mainly due to the fact that most of these enterprises were already founded as municipal ones. Fiscal reasons were clearly the decisive ones. In the beginning this was intended in a more defensive manner to keep the proceeds of the gasworks which were already mostly municipal property. Later on the profits to be expected from electricity supply became an additional argument for the municipalisation. For the representatives of a real municipal socialism this was pure fiscalism: only the owner had changed but not the methods of the capitalist private economy striving for maximum profits. This reproach did not worry the pragmatically thinking and acting mayors. Municipal trading was for them an integral feature of a good administration, and to cite a "Kathedersozialist", it was regarded as a cultural progress. When the Verein für Socialpolitik adressed the phenomenon of municipal trading this was already developed as far as possible. Certainly the Kathedersozialisten had prepared an ideological climate favourable to public intervention, the municipal socialism in the German manner evolved in the cities elicited by the forces of circumstance. Given the farreaching political powerlessness of the German socialists municipal socialism lost its political explosive force. It was no more than a catchy wording for a systematic and scientific administration of the city. 